\title{
Diagnosis “Autism” - from Kanner and Asperger to DSM-5
}

\author{
Meglena Achkova and Harieta Manolova*
}

\author{
Child Psychiatric Clinic "St. Nicholas", University Hospital "Alexandrovska”, Sofia, Bulgaria
}

\begin{abstract}
The authors make a synthesized overview of the evolution of the understanding of autism in historical context and a critical analysis of the development of diagnostic criteria in the spirit of the Diagnostic and Statistical Manual (DSM). Based on personal research and extensive clinical experience they put forward a number of debatable issues and own views about the nature of autistic disorder by outlining the trends and directions for future research. Discussed is the issue of "core" and "additional" symptoms of autism and the need for comparison of categorical and dimensional data when constructing empirical studies for the autistic population. The article contains reflections on the underlying impairment which, according to the authors, is a disturbance in the processing and integration of the incoming information, especially at the level of filtration of significant and insignificant stimuli and their linking into a mental sequence appearing at different levels and with varying degree of severity. Noted is the importance of the detailed assessment of mental functioning for early diagnosis and individualized targeting of the therapeutic efforts. In this regard is emphasized the need to search for a new paradigm in the methodology of future research on autism that would make possible the comparison of interdisciplinary results and identification of connections between the relevant scientific achievements. Thus it will be possible to identify trends that will bring us closer to revealing the etiology, perhaps will have an impact on the criteria for diagnosis and on the overall construction of future therapeutic strategies.
\end{abstract}

Keywords: DSM, "core"/"additional" symptoms, dimensional/categorical analysis, integration/filtration of information, interdisciplinary comparable studies.

\section{BRIEF HISTORICAL OVERVIEW}

The term "autism" was introduced in 1911 by the Swiss psychiatrist Eugen Bleuler when he described the social withdrawal behaviour as "turning inward" [1] into the own world and denial of contact. For a long time autistic disorder was considered a form of schizophrenia manifestation.

In 1943 based on observation of 11 cases Leo Kanner in the U.S. described a peculiar syndrome typical for the early childhood and called it "infantile autism". According to Kanner, the main symptoms are autistic isolation and desire for the preservation of sameness [2].

Independently from him, in 1944 the Austrian psychiatrist Hans Asperger published a description of 4 cases of children exhibiting what he called "autistic psychopathy". Asperger's work remained almost unknown in the U.S. and other English speaking countries until it was recommended in a publication of Lorna Wing in 1981 and translated into English in 1991 [1].

Both authors [1, 2] talk about features such as social withdrawal, stereotype movements and manipulations, resistance to changes and odd interests. In Kenner's group the children are generally

*Address correspondence to these authors at the Child Psychiatric Clinic "St. Nicholas", University Hospital "Alexandrovska", 1, G. Sofiiski Blvd., Sofia 1431, Bulgaria; E-mail: harieta.manolova@gmail.com with more severe retardation, they don't have a developed speech or it is limited and stereotypical. The children in Asperger's group are with well-developed speech, above average intelligence, peculiar interests, with psychomotor instability. Both authors distinguish autism from schizophrenia and Kanner noted that unlike the childhood schizophrenia where there are at least two years of normal development, the children with infantile autism seem to be born unable to connect normally with other people and respond accordingly to the situations [2, pp 248-249]. He comments that: "these children have come into the world with innate inability to form the usual, biologically provided affective contact with people, just as other children come into the world with innate physical or intellectual handicaps" [2, pp 250].

In 1966 Andreas Rett [3] reported about 22 girls who have exhibited a combination of severe autistic behaviours after the first 5-6 months of their life, during which they had a normal development. In 1999 Huda Zoghbi proved that Rett's syndrome is caused by a mutation in the MECP2 gene [4].

In 1968 Michael Rutter published the results of a comprehensive study of the scientific reports of autism and its symptoms, which approximates the modern-day diagnostic criteria described in the conventional classifications of diseases. He proposed to group the autistic symptoms into three main categories: lack of interest in communication; communication disorders (in qualitative rather than quantitative aspect); lack of imagination with manifestation of limited interests and 
ritualistic behaviours. Michael Rutter specified also that these symptoms occur before the third year after birth [5].

Research through the years showed that there are many variations of autistic disorder between the two groups as described by Kanner and Asperger. Lorna Wing (UK) introduced the term 'autistic continuum' which comprises the entire range of diverse forms and manifestations of autism; in 1979, based on an epidemiological study and clustering of the results, she described the "triad of impairments" typical for this group of disorders: impairments of social interaction (isolation, passivity/activity, oddities); impairments of communication (verbal and non-verbal); impairments of imagination and thinking (symbolic games, stereotypical repetitiveness, understanding of emotions) [6]. This is one of the most significant contributions to the clinical understanding of autism as a spectrum $-\mathrm{L}$. Wing believes that autism is a disorder of development and that the various manifestations, whether or not called "syndromes", are part of a single "spectrum" of interrelated disorders.

\section{DIAGNOSTIC CRITERIA FOR AUTISM IN THE DSM}

The American Psychiatric Association had not published separate criteria for autism until the year 1980; in the DSM-I (1952) [7] autism was defined as "psychotic reaction" and was coded under the "schizophrenic reactions in childhood" heading and the phrase "autistic behaviour" appeared in the description of childhood schizophrenia in DSM-II (1968) [8]. In DSM-III (1980) [9] for the first time were mentioned 6 criteria for diagnosing "infantile autism" relative to the early age (before $30^{\text {th }}$ month), disorders of the social communication and language, odd interests and fear of change, and for the first time was emphasized the distinction of autism from schizophrenia. In DSM-III-R (1987) [10] the diagnostic criteria for "autistic disorder" were grouped in three main areas of impairments (of social interaction; of communication and imagination; restricted repertoire of activities and interests), with a distinct reference to the onset "after the $36^{\text {th }}$ month". There the descriptions of behavioural peculiarities were already much richer, detailed and backed up with examples from the clinical practice facilitating the diagnosing and showing a much more complete picture of the autistic functioning. There was also an attempt to rank the severity of the disorder, as the items were ranked from the earlier age and more retarded to the older age and less severe cases. In DSM-III (1980) [9] for the first time was mentioned criteria for "childhood onset pervasive developmental disorder" (PDD), bringing forwards the notion of a "syndrome" and emphasising the pathology of the affects, bizarre movements, abnormal speech, hyper- or hyposensitivity and self-harm; also mentioned was the "asociality and lack of empathy". In DSM-III-R (1987) [10] was added a new diagnostic category of "pervasive developmental disorder not otherwise specified" and was clarified that disorders of the social interaction and communication are present, "but the criteria for autistic disorder, schizophrenia, schizotypal or schizoid personality disorder are not met". In DSMIV (1994) [11] the "pervasive developmental disorders" were characterized by disturbed social interaction, communication and peculiarities of behaviour, a "condition" and developmental delay were mentioned, including "autistic disorder, Rett's disorder, childhood disintegrative disorder, Asperger's disorder and disorder not otherwise specified". The presence of comorbidities was assumed and coded separately and the condition was distinguished from the schizophrenia; however, it was made clear that this did not preclude an individual with PDD developing schizophrenia later in life. In DSM-IV-R (2000) [12] the term "autism spectrum disorder" (ASD) was mentioned for the first time as synonymous to "pervasive developmental disorders", along with the term "atypical autism" as a synonym of "disorder not otherwise specified". In DSMIV (1994) and DSM-IV-R (2000) the symptoms of autistic disorder were classified still as a "triad of impairments": social interaction, communication and presence of restricted, repetitive, stereotyped patterns of behaviour. The presence of identical symptoms describing the disorder in both children and adults, along with the lack of clarifications associated with the prognosis was problematic. In principle, the DSM-IV targeted predominantly the professionals and accuracy of diagnosis by including five different disorders (autistic disorder, Rett's disorder, childhood disintegrative disorder, Asperger's disorder, pervasive developmental disorder not otherwise specified) grouped in the "pervasive developmental disorders" syndrome, which on one hand demonstrates the understanding of a common basic set of symptoms, and on the other hand introduces the idea of a wide variety and significant differences in the severity. DSMIV was a turning point in the elucidation of the single diagnostic category concept, which was reflected in the DSM-5 (2013) [13] as "autism spectrum disorder". Observed was an adding of symptoms such as sensory interests and strong aversions that have not been included previously as criteria in the DSM-IV [11, 12]; 
used was a two-dimensional model of the symptoms by combining the deficits in social interaction and communication into a single diagnostic category; the second category was related to "restricted, repetitive patterns of behaviour, interests, or activities" [13]. The symptoms must be present in the early stages of development (but may not be fully manifested until the social demands exceed the limited capacity or may be masked by learning strategies in later periods of life) i.e. a relaxing period was given for the onset of the disorder. In DSM-5 the differences in ASD were referred to a specific pattern of symptoms such as number, severity and progressive development; at the same time it is possible for the ASD to be diagnosed with other disorders. DSM-5 was more focused on descriptions of specificities and deficits in the functioning - i.e. it was focused at the services and assistance that the child and family should/may use. There was an emphasis on the hyper-sensitivity added criteria such as sensory interests and strong frustration upon sensory stimulation typical for the infancy. There was no separate criterion for the speech peculiarities, as it was considered non-substantial in children with ASD from the DSM-5 revision Committee and referred to another diagnosis - "social communication disorder" (SCD) [13], while the clinical experience showed major significance and specificity of this psychopathology when diagnosing. Coming up were issues relative to the emergence of the "new diagnosis SCD, which describes children with difficulties in the pragmatics of verbal and non-verbal communication, which leads to impaired social function" [14, pp 161]. The experienced clinicians are able to distinguish between language development disorders associated with preceding and accompanying psychomotor difficulties linked to spatial relations and autistic-type communication disorder. Since in the DSM-5 the severity of the disorder was determined through descriptions of dysfunctions and there was no precise definition of the various degrees, one may already notice attempts to provide extra "schemes" for categorization of the various levels of functioning while avoiding the reference to the individual IQ and the level of social adaptation, which may be relatively objectively measured with the standardized methods of the clinical psychology.

In the recently promoted experience for rating the severity [15] of autism spectrum disorder to the Criterion D specified in the DSM-5, where the two main groups of symptoms are together (related to the social interaction/communication and restrictive/repetitive behaviours), it is worth noting the referral of the severity to the problems of functioning with emphasis on the need of support/accompaniment and difficulties in regulating the behaviour, which is a major problem for the caregivers and is associated with the most common model for interventions - cognitivebehavioural approach. Under this approach, through a clear and unchanging structure of activities and daily routine and while relying on the natural tendency of the autistic individuals to display stereotypes, monotony and repetition, are "fixed" socially desirable behaviours and the pathological reactions such as strange rituals, routine actions and activities are "discontinued" or reduced as much as possible. In the proposed rating scale [15] for determination of the levels of severity of the disorder there were no precise criteria for measurement and evaluation of the degrees, which therefore leads to confusion and subjective description of behaviours and deficits. The idea of a single assessment toolkit in the diagnosis of autism has its positive aspects such as commensurability and comparability of data, but also its limitations in terms of the opportunities for new searches and identification of non-conventional results, i.e. it reduces the chances of discovering new regularities of the autistic functioning by analysing the intra-dimensional and intergroup relations in the psychic profiles of the autistic individuals in the various stages of mental development. There are also other specificities related to the source of information which in most of the toolkits (ABC, CARS, M-CHAT, CSBS-DP-IT-Checklist, ASQ, AQ, CAST, ADI, DISCO, ADOS, ASDS, GADS, ASDI, SRS ...) [16] are mainly based on the parental evidence of the progress and peculiarities in the behaviour of the autistic children in the various age stages. The parents' associations are important element of the support and sharing of the suffering, but they also have a powerful effect on the understanding and treatment of autism in the recent years, which effect is not always completely objective, as it is influenced by the natural process of emotional commitment. For example, parental descriptions of the "normal progress" in the early development and the "rupture" (often associated with a traumatic event or gradual "loss" of abilities) need objective scientific evidence and they are important element in guiding the etiological searches. In the present era of technology where filming is possible many families have a rich fund of recorded behaviours of autistic children from their earliest age. Our experience shows that when parents, aiming to showcase the 'normal' early development of their children, have shown us such 
footages, the avoidance of interaction and lapsing into autistic activities in the first months of life of the affected children has been apparent. The parental concerns are related to the determination of the severity, as the most insurance systems are based on it and it is the basis for determining the package of services (health, social, educational, financial ...) that autistic individuals use every day and throughout life. The caregivers also need such grading in order to organize their work and justify/quantify their commitment. It is this "demand" in particular that the new classification largely corresponds to, but the studies emphasize that for persons with diagnoses as per DSM-IV - Asperger's disorder or PDD-NOS, for those with higher IQ and for the female gender the risk of dropping out of the service system with the introduction of DSM-5 is the highest [14, pp 161].

In general, the changes in DSM-5 should lead to a more reliable diagnosing, with prognoses for the individual severity and the associated preconditions for improvement of the condition, but due to the lack of precise criteria for determining the degree of dysfunction, one will have to rely on the opportunity to examine carefully the detailed history of the child and family, to draw information from many different sources, simultaneously with the clinical observation, making the diagnosis largely dependent on the personality and experience of the respective professional. For this reason still the greatest impact on the quality and objectivity of the diagnostic process has the longer observation, the regular reassessments of the functioning and the team - diagnosing.

\section{DISCUSSION}

Before comparing the evolution of criteria for diagnosis of autistic disorder in DSM it would be appropriate to analyse what advantages the new classification is giving us. This is clearly highlighted in the name itself - Diagnostic and statistical manual, which is based on statistics and scientific research (also validated with statistical significance of the results), i.e. it rather gives information on the frequency of the disorder in the population and specifies uniform criteria for determining the "cases" when forming groups for scientific research, therapy and referrals to specialists. The DSM cannot act as substitute of the experience and flair to the fine details of the complex and diverse clinical picture of the autistic disorder. Expressed in medical terms - it allows a sufficient description, in terms of its validity and specificity, of the "clinical picture" of a given disorder or syndrome. The term "disorder" is more appropriate because it saves us the highly rigid boundaries of concepts such as "disease" and "nosological unit", for which the classical scholars have long ago determined precise requirements: identification of etiology, pathogenesis, clinical picture, course and outcome. Unfortunately, rarely the outlined clinical pictures in psychiatry can meet these requirements. What do the updates of diagnostic classifications in psychiatry give us or at least strive to give us? We believe that the gathering of large enough aggregate of "cases" identified as such by uniform criteria results in a group of basic - "core" symptoms that have already been recognized as a result of multiple series of scientific and clinical researches. Along with the "core" symptoms exists a group of "additional" symptoms that may extend the diagnostic boundaries of the disorder. Hence, this leads to establishment of the "autism spectrum" concept without taking into account the pathogenetic factors, which is not useful for the future scientific research related to autism. For the sake of searching possible genetic and psychophysiological factors in the autistic disorder, one should select very specific patients, not every case falling somewhere within the spectrum. This means - carriers of the "core" symptoms that have been sufficiently psychologically analysed in term of affected areas and degree of implication in the various areas. In addition to the categorical question "what?" is required also the dimensional criterion "how much?" Only then follow the questions "how?" (how does the disorder occur pathogenesis) and "why?" - etiology. This will help not only the researchers, but also the professionals in their educational - therapeutic efforts, knowing where to focus and what they can rely on.

Indicative of the rigidity of such a classifier is also the fact that despite the advent of autism in the scientific literature in 1943, it was not included as a separate diagnostic category before the third edition of the DSM published 37 years later. We notice similar delay also in DSM-IV (published in 1994, revised in 2000) containing a description of the typical "triad of impairments" identified on the basis of the study of Lorna Wing and Judith Gould in 1979. Ardent debates between the professionals and multitude of scientifically unproven methods claiming to be discoveries of the causes and "treatment" of autism stem precisely from the actual lack of information on the pathogenesis, lead to therapeutic frustration and outline a pessimistic prognosis of life-time condition.

Like with the understanding of schizophrenia, in the case of autism there is also a transition from the idea of 
"fragmentation" to the idea of "unification" within a single general disorder, by including a number of disturbances such as Rett syndrome for example, which is connected rather to a dementia process than to an autistic dysfunction, or the childhood disintegrative disorder, where there is a disintegration of something already existing, while in the case of autism obviously it is about an impossibility to build something. The varying degree of impact caused by the disorder also leads to the hypothesis that autism is caused by a combination of genetic factors, wherein the greater fragility leads to greater severity of the disorder, while the more persistent genetic structures "lighten" the damage caused. The typical "disharmony" observed by the clinical psychologists in the mental profiles of autistic individuals shows clearly the difficulty to build and progressively "fill" the gaps in the different stages and aspects of psychological development, because of the difficulties to make connections and identify relationships between objects, subjects and events. Comparison of the psychic functioning profiles of the autistic children in early childhood (up to 24 months of development) in personal research [17] shows interesting findings in the dimensional analysis of individual and intra-group relations between the studied indicators of development. Outlined are some typical characteristics of the autistic cognitive, sociocommunicative, socio-emotional and sensory-motor functioning different from those of the normative group of healthy children and of those raised in a situation of social and emotional deprivation [18] (in specialized institutions for abandoned children) or with Down syndrome [19]. Clinical practice shows that such detailed psychological assessment supports early diagnosis, facilitates the construction of individualized and most effective intervention programs, and outlines clinical subgroups associated with different severity of impact by the disorder. Even in the least severe cases, a lack of sensitivity to the synthesis and literal transfer of information is observed, along with extreme "cyclic focus" in a narrow field, subject of endless studies, which obviously leads to comfort and pleasure of "putting in order" the cognitive construct. Obviously, the variety of relationships, emotions and context destabilize, as they influence and change the established cognitive order that autistic individuals stubbornly oppose to the chaos of different perceptions, which they lose orientation in and that threatens to destroy even the smallest "islands" of understanding and knowledge that they have so laboriously managed to build.
Based on the bibliographic sources [1-20] and the clinical experience we may conclude that:

- $\quad$ Autism is part of a broader spectrum of disorders with varying severity of deficits and level of psychic functioning. With yet unidentified genetic modality and multiplicity of additional factors accompanying the individual phenotype, whose presence and/or accumulation is probably related to the severity of the disorder.

- $\quad$ All differentiated in the diagnostic classifications disorders from the autistic continuum are characterized by a general disorder of the psychic development and by substantial delay of the communication and language. Obviously, it is a complex problem related to the ability to perceive, filter and integrate information.

Never was including in the criteria for diagnosis the feature uniting all cases, namely the inability to integrate information - from level of perception to formation of a concept and understanding the incoming information by its filtering in accordance with its significance. Ontogenetically the psychic development goes from the simple to the complicate, but autistic individuals are not able to distinguish between what is simple and what is complicate. Autistic behaviour is a defensive mechanism of the psyche, helping to prevent a total collapse in the influx of perceptions because of their difficult reduction and selection, which prevents the synthesis and symbolisation - foundation of thinking and understanding the world around us. An understanding, which for us is precisely a continuous (to a large degree automated) rationalization of connections and relationships, but for the autistic individuals is a major deficit, a source of strong fears unexplainable to us, and endless studies of detail which is meaningless to us, cyclic focus on one elementary sensation, infinite repetition of the same actions and/or phrases that are obviously a safeguarding partial attempt - a source of security and temporary peace in this confused and incomprehensible, hence so scary autistic world.

There are two basic approaches for conceptualization of the structure of autism that may be defined as dimensional and categorical model. The dimensional approach considers autism as a continuous variable, implying that there are one or more dimensions of autism, within which all individuals of the population may be located - i.e. the individuals are discriminated on the basis of the severity of the 
disorder (DSM-5), unlike the categorical approach, which supports the idea that there are many forms of autism (DSM-IV). The credibility of a study, which is aimed at identifying the most effective diagnostic and treatment methods (such as the purpose of the DSM) increases when the basic structure of the target pathology is known [20], but unfortunately this still does not apply to the autistic disorder. Vacillation in either extreme when constructing the research of the autistic population is not helpful and it is therefore necessary to strike a balance using a parallel analysis of simulated comparison of categorical and dimensional data. Such an approach would have value also in the interpretation of the results, given that "the categorical latent structure is generally indicative of the presence of a discrete etiological source (such as a particular gene, a nervous system disorder, an environmental stressor, or a specific interaction of these variables), while the dimensional structure means that the phenomenon is multiply determined and has an additive or graded etiology" [20].

The unusually quick development of technology has created a new era in biology - the era of molecular biology. Increasingly complex microsystems in the brain cells have been revealed - membrane, electrolyte, enzyme, mediatory, receptor systems and their significance to the brain functioning and, in particular, to the psycho-physiological mechanisms involved in the mental activity. Revealed are also some genetic and epigenetic (regulatory) mechanisms involved in the development and functioning of the nervous system. This implies that in the future the diagnostic criteria for autism will no longer be directed mainly to descriptions of behaviour or level of functioning. They will be also based on neurological (focusing on the transmitters), genetic (associated with the gene combinations) medical research and detailed dimensional assessments of the functioning with identifying typical group characteristics and individual intra-profile specificities. The therapy, which is currently limited to intensive treating of the behaviour by a multitude of repetitions and support to the desired responses with suppression of undesirable behaviours and attempts for generalization of what is learned in different social contexts, without ultimate overcoming of the autism symptomatic, in the future will probably include more neuropsychological and neurogenetic results and opportunities for interventions.

As it had often happened, the detection of the cause of autistic behaviour will contribute to the better knowledge and understanding of the psychic functioning of the healthy person in general. In all branches of science nowadays are carried out uncoordinated studies resulting in "flood" of information that remains insufficiently comparable. Scientific achievements look like the "white spots" in the mental functioning of autistic individuals, and the scientific research becomes an autistic occupation without strong connection with the total stream of information. It is necessary to unite the efforts of science in all its subdomains and create a single base of comparable data from multicentre studies. This will permit us to systematize the available information, to establish links and relationships between the multitude of known and studied factors that influence the formation and growth of the human being and are deregulated by autistic children.

\section{REFERENCES}

[1] Rau JD. Is it autism? J Contemp Pediatr 2003; 20: 54-82 [accessed 2 March 2014]. Available from: http://www. mm3admin.co.za/cms/cpd/articles/

[2] Kanner L. Autistic disturbances of affective contact. J Nerv Child 1943; 2: 217-50.

[3] Schopler E. On confusion in the diagnosis of autism. J Autism Childhood Schizophr 1978; 8: 137-8. http://dx.doi.org/10.1007/BF01537862

[4] Amir R, Van den Veyver I, Wan M, Tran C, Francke U, Zoghbi $H$. Rett syndrome is caused by mutations in X-linked MECP2, encoding methyl-CpG-binding protein 2. J Nat Genet 1999; 23:185-8.

http://dx.doi.org/10.1038/13810

[5] Rutter M. Concepts of autism: a review of the research. $J$ Child Psychol Psychiatry 1968; 9: 1-25.

http://dx.doi.org/10.1111/j.1469-7610.1968.tb02204.x

[6] Wing L, Gould J. Severe impairments of social interaction and associated abnormalities in children: epidemiology and classification. J Autism Dev Disord 1979; 9: 11-29. http://dx.doi.org/10.1007/BF01531288

[7] American Psychiatric Association. Diagnostic and Statistical Manual: Mental Disorders. Washington, DC: Author, Mental Hospital Service 1952.

[8] American Psychiatric Association. Diagnostic and Statistical Manual: Mental Disorders ( $2^{\text {nd }}$ ed.). Washington, DC: Author 1968.

[9] American Psychiatric Association. Diagnostic and Statistical Manual of Mental Disorders ( $3^{\text {rd }}$ ed.). Washington, DC: Author 1980.

[10] American Psychiatric Association. Diagnostic and Statistical Manual of Mental Disorders. DSM-III-R. Washington, DC: Author 1987.

[11] American Psychiatric Association. Diagnostic and Statistical Manual of Mental Disorders (4 ${ }^{\text {th }}$ ed.). Washington, DC: Author 1994.

[12] American Psychiatric Association. Diagnostic and Statistical Manual of Mental Disorders ( $4^{\text {th }}$ ed. - Text revision). Washington, DC: Author 2000.

[13] American Psychiatric Association. Diagnostic and Statistical Manual of Mental Disorders ( $5^{\text {th }}$ ed.). Washington, DC: Author 2013.

[14] Lohr WD, Tanguay P. DSM-5 and Proposed Changes to the Diagnosis of Autism. J Pediatr Ann 2013; 42: 161-6. http://dx.doi.org/10.3928/00904481-20130326-12 
[15] Carpenter L. DSM5 Autism Spectrum Disorder - Guidelines \& Criteria Exemplars. Medical University of South Carolina Feb. 2011.

[16] Volkmar FR, Siegel M, Woodbury-Smith M, King BR, McCracken J, State M. AACAP - CQI. Practice parameter for the assessment and treatment of children and adolescents with autism spectrum disorder. J Am Acad Child Adolesc Psychiatry 2014; 53: 237-57. http://dx.doi.org/10.1016/j.jaac.2013.10.013

[17] Manolova H. Early psycho diagnosis of children with PDD childhood autism for the needs of clinical practice. In: BAPID

Eds. Childhood autism - therapeutically approaches and including education. Sofia: Phare BG 2003/004-937.01.04, 2008; pp. 15-33.

[18] Manolova H. Effect of Granny program in Bulgaria on the early child development. New York: Spence - Chapin Services to Families and Children 2010.

[19] Nader-Grosbois N, Milusheva R, Manolova H. Profils multidimensionnels de jeunes enfants trisomiques bulgares. J Psy Fra 2006; 51: 391-11.

[20] Broman-Fulks JJ, Hill RW, Green BA. Is perfectionism categorical or dimensional? A taxometric analysis. J Pers Assess 2008; 90: 481-90.

http://dx.doi.org/10.1080/00223890802248802

\section{DOI: http://dx.doi.org/10.6000/2292-2598.2014.02.02.4}

(C) 2014 Achkova and Manolova; Licensee Lifescience Global.

This is an open access article licensed under the terms of the Creative Commons Attribution Non-Commercial License (http://creativecommons.org/licenses/by-nc/3.0/) which permits unrestricted, non-commercial use, distribution and reproduction in any medium, provided the work is properly cited. 